\title{
COMMENTARY
}

\section{The case for frugal default options in patient-doctor communication}

\author{
Angela Fagerlin $\mathrm{PhD}$ \\ Ann Arbor VA HSR\&D Center for Practice Management and Outcomes Research; Division of General Internal Medicine, University of Michigan; \\ Center for Behavioral and Decision Sciences in Medicine, Ann Arbor, Michigan, USA \\ doi:10.1111/j.1365-2753.2010.01404.x
}

Bogdan-Lovis and Holmes-Rovner [1] make a compelling case for using frugal default options to help patients and doctors make decisions, while also reducing health care costs, in situations defined by medical equipoise (reference). Their proposal is that in medical conditions in which the available treatment options do not differ in their clinically effectiveness (e.g. result in equivalent survival rates) doctors should recommend the alternative that requires the least resources. They do, however, emphasize that while the frugal option should be offered first, patients and their doctors must have the ability to 'override' the default if doing so better reflects the patients' values and preferences.

This proposal has considerable merit. Patients often do not want to make these difficult decisions and often look to their doctor to help make the decision [2,3]. If the arguments for different treatment options really embody equipoise, doctors need some other justifiable criteria upon which to base their recommendation, especially given the fee-for-service environment which often rewards doctors for providing the most aggressive, costly treatments.

To determine the feasibility of this approach, however, we must ask (at least) two questions. First, is implementing a frugal default option likely to influence patient decision making and, thus, reduce health care costs? If yes, is the frugal default option the best method for balancing a respect for patient autonomy and health care costs? I will address each question in turn.

The judgement and decision making literature is filled with examples where the default option strongly influences people's decision making [4-7]. In examining the rates of organ donation in Europe, Johnson and Goldstein compared the rates of countries that had opt-in versus opt-out defaults [6]. The four countries that used opt-in defaults had significantly lower rates (range: 4.25$27.5 \%$ ) than those countries that had opt-out defaults (range: 85.9 99.98). In the domain of living will completion, one study found that older outpatients (65 years and older) who were given living will forms that were written with the default option being to receive treatment wanted more treatment in 21 of 22 different care decisions (and was significantly higher in seven of those decisions) than did those receiving living will forms that either did not contain a default option or that had the default option of not receiving treatment [4]. Similar strong effects have been found in decisions about flexible spending accounts [8], and mandated flu vaccinations for all health care workers [8].

There have been a number of explanations for the default bias. Choosing the default option may minimize decisional conflict and reduce the cognitive effort required to make a choice [9]. Additionally, many people view default options as recommendations made by a policy maker or authority figure $[7,8]$ and thus attribute more validity to them. This would be particularly true in cases in which the authority figure is a trusted figure [8]. These explanations suggest that if a frugal default option was implemented it would be a powerful influence on patients' decision making and the treatments they ultimately received. This, in turn, would likely result in decreased health care costs.

Now to turn to the second question, what are the implications of this policy? Is the frugal default option the best option for balancing patient care and health care costs? In many cases I agree that the default option would allow for maintaining patient autonomy, would help patients make difficult decisions, would provide a balance to the biases inherent in the fee-for-service approach to medicine, and would result in lower health care costs. The frugal default option is most appropriate for situations in which the distribution of preferences is normal. It is, however, less appropriate for situations in which $50 \%$ of patients love treatment option 1 and are vehemently opposed to option 2 while the other $50 \%$ are equally strong in their opposition to treatment option 1 and strongly prefer option 2 . Thus, the implementation of the frugal default strategy would need to depend on whether preferences are, across a population, consistently uncertain or dramatically variable for any particular treatment decision.

Furthermore, when the frugal default option is recommended by a trusted health care provider, it is likely that a non-trivial number of people are likely to accept a treatment choice that is different from the choice they would have made had they not been presented with a default option. This could be beneficial to many patients - having less invasive treatment can result in quality of life improvements and having someone else make a recommendation could reduce some of the burden on the decision-making process by making such options more palatable. However, because the likelihood of this occurring is significant, it is still critical that patients are informed of both choices and that they receive a decision aid that describes the evidence regarding the risks and benefits of all relevant treatment options. In addition, when describing the frugal default option to patients, it is important that patients not only understand but feel enabled to make alternate choices if they so prefer.

Bogdan-Lovis and Holmes-Rovner make a courageous argument for changing how doctors advise patients in situations that have clinical equipoise. Many patients are reluctant to make final treatment decisions and prefer help from their doctors. By having a justifiable default option that anchors discussions of treatments with doctors, patients may experience less stress and anxiety in their decision making. Furthermore, if the least costly intervention becomes the default, patients may receive less invasive treatments, an outcome which could lead to significant reductions in health care costs while simultaneously improving psychological (and perhaps even physical) outcomes for the patient. 
Our country needs to rethink our health care system and find ways in which we can reduce health care costs while also maintaining the care of our citizens. Bogdan-Lovis and Holmes-Rovner have proposed a method that is both feasible and which will preserve of patient autonomy, yet significant in its potential system-wide implications. It is critical that policy makers, doctors and social scientists strongly consider the recommendations made in their article.

\section{Acknowledgements}

The author is very grateful for the helpful comments she received from Brian Zikmund-Fisher on a previous version of this paper. The opinions expressed in this article are solely those of the author and are not reflective of the opinions of the Department of Veterans Affairs.

\section{References}

1. Bogdan-Lovis, E. (L.) \& Holmes-Rovner, M. Prudent evidence-fettered shared decision making Journal of Evaluation in Clinical Practice, 16 (2), 376-381.
2. Schneider, C. E. (1998) The Practice of Autonomy: Patients, Doctors, and Medical Decisions. New York: Oxford University Press.

3. Ubel, P. A. (2002) 'What should I do, doc?': some psychologic benefits of physician recommendations. Archives of Internal Medicine, 162, 977-980.

4. Kressel, L., Chapman, G. \& Leventhal, E. (2007) The influence of default options on the expression of end-of-life treatment preferences in advance directives. Journal of General Internal Medicine, 22, 10071010.

5. Samuelson, W. \& Zeckhauser, R. (1988) Status quo bias in decision making. Journal of Risk and Uncertainty, 1, 7-59.

6. Johnson, E. \& Goldstein, D. (2003) Do defaults save lives? Science, 302, 1338-1339.

7. Johnson, E. \& Goldstein, D. (2004) Defaults and donation decisions. Transplantation, 78, 1713-1716.

8. Halpern, S. D., Ubel, P. A. \& Asch, D. A. (2007) Harnessing the power of default options to improve health care. New England Journal of Medicine, 357, 1340-1344.

9. Chapman, G. B. \& Elstein, A. S. (2000) Cognitive processes and biases in medical decision making. In Decision Making in Health Care: Theory Psychology and Applications (eds G. B. Chapman \& F. A. Sonnenberg), pp. 183-210. Cambridge: Press Syndicate of the University of Cambridge. 